\title{
The Distances in the Stable Systems Due to the Virial Theorem
}

\author{
Hasan Arslan \\ Physics Department, Bingöl University, Bingöl, Turkey \\ Email: hasanarslan46@yahoo.com
}

Received January 30, 2013; revised March 4, 2013; accepted March 11, 2013

Copyright (c) 2013 Hasan Arslan. This is an open access article distributed under the Creative Commons Attribution License, which permits unrestricted use, distribution, and reproduction in any medium, provided the original work is properly cited.

\begin{abstract}
The virial theorem is written by using the canonical equations of motion in classical mechanics. A moving particle with an initial speed in an n-particle system is considered. The distance of the moving particle from the origin of the system to the final position is derived as a function of the kinetic energy of the particle. It is thought that the considered particle would not collide with other particles in the system. The relation between the final and initial distance of the particle from the origin of the system is given by a single equation.
\end{abstract}

Keywords: Virial Theorem; Distance; Stable System

\section{Introduction}

The calculations on the virial theorem are done to obtain an equation explaining the relations between the kinetic energy and potential energy of a particle and an equation describing the maximum distance travelled by this particle in a stable system. In Section 2, these equations are obtained due to the calculations. The previous works are searched. It was realized that the Equations (13) and (16) are first presented in this study. The calculations done in this study will be useful in astronomy. In Section 3, the results of this study are discussed.

\section{Calculations}

The virial theorem gives the general equation between kinetic energy and potential energy of a stable system of n particles bound by potential forces. R. J. E. Claussius formulated the virial theorem in 1870 after twenty years long study [1]. The word "virial" comes from the Latin word vis which means "force" or "energy". The reader who likes to learn more about the virial theorem is recommended to see the book [2]. The article [3] includes an extensive research on the virial theorem. The article [4] includes a search about finite deformations in the boundary conditions.

For system of masses at points $r_{i}$ the equations of motion are;

$$
\dot{\vec{p}}_{i}=\vec{F}_{i}
$$

A quantity $G$ is defined in [5] as:

$$
G=\sum_{i} \boldsymbol{p}_{i} \cdot \boldsymbol{r}_{i}
$$

The time derivative of this quantity is

$$
\frac{\mathrm{d} G}{\mathrm{~d} t}=\sum_{i}\left(\frac{\mathrm{d} \boldsymbol{p}_{i}}{\mathrm{~d} t} \cdot \boldsymbol{r}_{i}+\boldsymbol{p}_{i} \cdot \frac{\mathrm{d} \boldsymbol{r}_{i}}{\mathrm{~d} t}\right)
$$

where

$$
\begin{aligned}
& \sum_{i} \boldsymbol{p}_{i} \cdot \frac{\mathrm{d} \boldsymbol{r}_{i}}{\mathrm{~d} t}=\sum_{i}\left(m \dot{\boldsymbol{r}}_{i}\right) \cdot \dot{\boldsymbol{r}}_{i}=m \dot{r}_{i}^{2}=2 T \text { and } \\
& \sum_{i} \dot{\boldsymbol{p}}_{i} \cdot \boldsymbol{r}_{i}=\sum_{i} \boldsymbol{F}_{i} \cdot \boldsymbol{r}_{i}
\end{aligned}
$$

In Equations (3) and (4) $T$ is the kinetic energy, $m$ is the mass of the particle, $p$ is the momentum related to the particle, $F$ is the force acting on the particle, and $r$ is the distance vector of the particle. We are going to obtain the virial theorem from the Equations (3) and (4) as below.

For a bounded quantity $G$, in a time interval of $\tau$, one can write:

$$
\frac{1}{\tau} \int_{0}^{\tau} \frac{\mathrm{d} G}{\mathrm{~d} t} \mathrm{~d} t=\frac{1}{\tau}(G(\tau)-G(0))=0
$$

and

$$
\frac{1}{\tau} \int_{0}^{\tau} \frac{\mathrm{d} G}{\mathrm{~d} t} \mathrm{~d} t=2 T+\sum \vec{F}_{i} \cdot \vec{r}_{i}
$$

if the momentum is periodic. Then we get the virial theorem as 


$$
2 T=-\sum_{i} \vec{F}_{i} \cdot \vec{r}_{i}
$$

Writing $T$ as

$$
T=\frac{p^{2}}{2 m}
$$

and dropping the summation sign, the Equation (7) is written in the form:

$$
\frac{p^{2}}{2 m}=-\frac{1}{2} \vec{p} \cdot \vec{r}
$$

In [6] the force is related to the Hamiltonian by

$$
\left|\dot{\boldsymbol{p}}_{i}\right|=-\frac{\partial H}{\partial q_{i}}
$$

The scalar product $\boldsymbol{p} \cdot \boldsymbol{r}$ has to result in a scalar quantity. Therefore, the vector $\boldsymbol{r}$ changes to the distance $r$ in the following equations. By using this, the Equation (9) is written as

$$
\partial H=\frac{\boldsymbol{p}^{2}}{m q} \partial q
$$

Here, the notation $\partial$ is used as the differential d. Integrating this equation we get

$$
\int \mathrm{d} H=\int \frac{\boldsymbol{p}^{2}}{m q} \mathrm{~d} q \Rightarrow H=\int \frac{\boldsymbol{p}^{2}}{m q} \mathrm{~d} q
$$

For a particle moving with constant speed and using $H$ $=E=T+V$ and $q=r$ one can integrate from $r_{0}$ to $r$ to find the relation between kinetic energy $T$ and potential energy $V$ in time averaged values as

$$
V=T\left(-1+2 \ln \frac{r}{r_{0}}\right)
$$

For circular motion the equation is

$$
m a=\frac{m v^{2}}{r}=G \frac{M m}{r^{2}}
$$

In this case, the Equation (12) gives the correct classical gravitational potential energy if we integrate it from $r=\infty$ to $r=r-r_{0}$;

$$
H=\frac{G M m}{r-r_{0}}
$$

If we relate to this study, $M$ is the mass of the set of particles in the system and $m$ is the mass of the particle under consideration. The Equation (13) can be written as

$$
r=r_{0} \mathrm{e}^{E / 2 T}
$$

This is the equation for the kinetic energy $T$ of a particle in a stable system at distance $r$ from the origin of the system. $E$ is the total energy of the particle, and $r_{0}$ is the initial distance of the particle from the origin of the system.

\section{Conclusion}

The Equation (16) derived in this study explains that the distance of a moving particle in a stable system has a maximum distance $r$ if it remains in the system. The maximum distance must be the distance from the origin of the system to the outer surface of the system at most. When something moves away from the distance to the outer surface, it is out of the system. In other words, it can be said that; when this is reached, the stability of the system is destroyed or the travelled body is not a member of the system. The Equations (13) and (16) are first derived here comparing to the previous works.

\section{Acknowledgements}

I would like to thank to Prof. Dr. Amirullah Mamedov for his valuable discussion.

\section{REFERENCES}

[1] G. W. Collins II, "The Virial Theorem in Stellar Astrophics," 2003.

http://ads.harvard.edu/books/1978vtsa.book/bookfnt.pdf

[2] S. L. Shapiro and S. A. Teukolsky, "Black Holes, White Dwarfs and Neutron Stars,” John Wiley, New York, 1983. doi:10.1002/9783527617661

[3] J. F. Ganghoffer, "On the Virial Theorem and Eshelby Stress," International Journal of Solids and Structures, Vol. 47. No. 9, 2010, pp. 1209-1220. doi:10.1016/j.ijsolstr.2010.01.009

[4] R. Sunyk and P. Steinmann, "On Higher Gradients in Continuum-Atomistic Modeling,” International Journal of Solids and Structures, Vol. 40, No. 24, 2003, pp. 6877 6896. doi:10.1016/j.ijsolstr.2003.07.001

[5] H. Goldstein, “Classical Mechanics,” Addison-Wesley Publishing Company, Inc., Reading, 1959.

[6] S. T. Thornton and J. B. Marion, "Classical Dynamics of Particles and Systems," Thomson Learning, Belmont, 2004. 